\title{
Behavior of Full-Scale Porous GFRP Barrier under Blast Loads
}

\author{
D. Asprone, ${ }^{1}$ A. Prota, ${ }^{1}$ G. Manfredi, ${ }^{1}$ and A. Nanni ${ }^{1,2}$ \\ ${ }^{1}$ Department of Structures for Engineering and Architecture, University of Naples "Federico II", 80125 Naples, Italy \\ ${ }^{2}$ Department of Civil, Architectural and Environmental Engineering, University of Miami, Coral Gables, FL 33124, USA \\ Correspondence should be addressed to D. Asprone; d.asprone@unina.it
}

Received 13 May 2015; Accepted 11 August 2015

Academic Editor: Osman Gencel

Copyright (c) 2015 D. Asprone et al. This is an open access article distributed under the Creative Commons Attribution License, which permits unrestricted use, distribution, and reproduction in any medium, provided the original work is properly cited.

This research paper is part of the SAS (Security of Airport Structures) Project funded by the European Programme for Critical Infrastructure Protection, whose objective was to develop and deploy a fiber reinforced polymer (FRP) fencing system intended to protect airport infrastructures against terrorist acts. In the paper, the efficacy of the proposed glass FRP discontinuous (porous) barrier under blast loads is presented by showing the results of the blast test campaign conducted on full-size specimens with a focus on the reduction of the blast shock wave induced by the barrier. A simplified model predicting the reduction of the shock wave beyond the barrier is proposed and validated via the experimental data obtained in the project.

\section{Introduction}

Recent terrorist acts have contributed to change of the design approach to critical infrastructures; in fact, malicious disruptions, blasts, or impacts have unfortunately become part of the possible load scenarios that could act on constructed facilities during their life span. Consequently, specific protection interventions are introduced to minimize disruptive effects, guarantee the safety of the occupants of a facility, and, to the extent possible, maintain the functionality of a facility. Hence, highly dynamic loading conditions represent nowadays a fundamental challenge in structural engineering as critical buildings and infrastructures need to resist extreme loads events that can occur during their lifetime as a result of natural and man-made hazards (e.g., explosions, collisions, and severe earthquakes). As a corollary, there is a growing interest in the scientific and practicing communities to design and/or assess protection systems to reduce the vulnerability of critical infrastructures (e.g., shelters and barriers). With this objective, a research program named Security of Airport Structures (SAS) was undertaken and completed by a consortium of European entities led by the research center AMRA (http://www.amracenter.com/), in order to (a) design and validate a protection barrier intended to deter malicious actions against critical airport infrastructures carried out by ecoterrorists and (b) mitigate the effects of blast events on protected targets.

A porous glass fiber reinforced polymer (GFRP) barrier was designed and its components were subjected to mechanical tests. The material of choice for this barrier system became GFRP and not steel or steel reinforced concrete, as widely used in practical engineering because of the necessity of maintaining radiotransparency without interference with airport radio communications. To assess this property radiotransparency tests of the barrier were also conducted in anechoic chamber [1]. Furthermore, a blast test campaign was performed on full assemblies in order to validate the capability of the system in withstanding blast loads and protecting a target placed beyond it by reducing the effects of the incident blast shock wave.

Mitigation techniques used for other extreme events such as earthquakes could be adopted to resist blast loads [2], and fiber reinforced polymer (FRP) composites comprise a promising strengthening solution in the form of an externally bonded system [3]. Conversely, using FRP for the construction of barriers or similar security has not yet been explored [4] even though glass FRP (GFRP) porous barriers have been identified as a possible measure to prevent malicious disruptions, provide a standoff distance in case of blast actions, and reduce the consequences of an impact. 
According to the end-user requirements, the main objective of the research project was to develop and deploy a structural fencing system able to protect VHF omnidirectional range (VOR) stations against malicious actions consisting of intrusion and/or blast loads of relatively small explosive charges placed in the vicinity or in contact with the barrier. A critical feature of any protective barrier for this type of facility is radiotransparency, which is necessary to avoid any disturbance to radio communications of specific frequencies. Hence, to achieve such goals, a discontinuous (porous) barrier composed by GFRP and precast concrete elements reinforced with GFRP bars was designed to take advantage of electromagnetic and mechanical properties of composites. The proposed barrier provides protection through two contributions. First, its geometrical and mechanical characteristics ensure protection against intrusions and blast loads. Second, its shape provides an attenuation of the blast shock wave, adding some level of additional protection for facilities located beyond it. The end-user specified the design charge for the blast action equal to $5 \mathrm{~kg}$ of commercial explosive, intended to represent the possible disruptive action of an ecoterrorist attacking radio communication infrastructures. Given the amount of explosive as a fixed parameter, in addition to contact charge configuration employed in third test, the first two tests investigated two different standoff distance configurations aiming at reproducing the cases of prevented direct access to the barrier.

The objective of this paper is to describe some results of the blast test campaign conducted on full-size specimens of the proposed barrier and to discuss their effectiveness in mitigating blast shock waves. A simplified model is also proposed to predict the reduction of the peak pressure due to the porous barrier; an example of the computational procedure is described in Appendix. Numerical tests, using the applied elements method, were also conducted, providing further details about the structural behavior of the barrier, and are presented in [5]. These analyses were used to validate the forces acting on the barrier and the structural response of the pipes.

\section{Materials and Methods}

2.1. Geometry and Mechanical Properties of the Barrier. The barrier is composed of vertical GFRP pipe elements secured in a precast reinforced concrete pedestal base. Each individual pipe is not connected to the adjacent ones to avoid the domino effect in case of failure. Each pipe has an external diameter of $85 \mathrm{~mm}$ and a wall thickness of $5.5 \mathrm{~mm}$. Pipes are spaced $75 \mathrm{~mm}$ on centers. The GFRP pipe wall is a typical pultruded product with an internal core reinforced with unidirectional E-glass fibers (to provide strength and stiffness in the longitudinal direction) and two external layers reinforced with a mat of E-glass chopped fibers (to facilitate manufacturing and allow for a resin rich surface). Both core fibers and mats are impregnated with a polyester resin.

The concrete base and the GFRP pipes are $0.5 \mathrm{~m}$ and $2.5 \mathrm{~m}$ high, respectively. The barrier is represented in Figure 1. The concrete base is made of interconnected precast segments

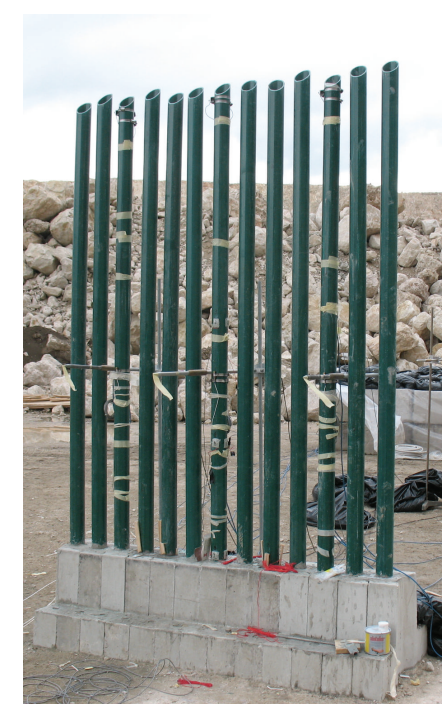

Figure 1: Prototype of the barrier.

(dry joint, male-female connection to provide shear interlock) having a thickness of $150 \mathrm{~mm}$. Each segment has an inverted $\mathrm{T}$ cross section, whose web and flange are $0.3 \mathrm{~m}$ and $0.7 \mathrm{~m}$ wide, respectively. The overall height and flange thickness are equal to $0.5 \mathrm{~m}$ and $0.2 \mathrm{~m}$, respectively.

One pipe is installed in each precast concrete segment in a $0.3 \mathrm{~m}$ deep, $100 \mathrm{~mm}$ diameter hole centered on the web of the precast element. The interspace between pipe and concrete is filled with a no-shrink cementitious mortar. Two additional holes for tie anchors are present in the flanges of each segment. Two GFRP bars can be inserted through these holes and secured with cementitious mortar to create a deep foundation system. The diameter and depth of these GFRP tie bars have to be determined based on ground characteristics.

The GFRP's mechanical behavior was investigated for a quality control process and to collect data that will be used in performance modeling (together with high strain-rate properties) not discussed herein. To this aim, coupon tests were conducted on flat GFRP specimens taken from the pipe to evaluate their direct tension properties [5]. Two typologies of specimens were tested:

(i) Integral one, composed of the internal core and the external layers.

(ii) Peeled one, composed only of the internal core (after machining the surface layers).

All the specimens exhibited a linear behavior up to failure. Table 1 shows the results derived from such tests.

Four-point bending tests were also performed on five pipe specimens to investigate their flexural behavior. This type of test is complementary to the tensile test on coupons in terms of quality control. The data were processed to obtain moment-curvature relationships, and those slopes indicate the flexural stiffness of the tested pipes. An average value for the flexural stiffness of $41.2 \mathrm{kNm}^{2}$ was obtained. A global Young's modulus was obtained that combines both tensile and compression contributions. To separate them and determine 
TABLE 1: Summary of the results of tensile failure tests on GFRP specimens.

\begin{tabular}{lcccc}
\hline & $\begin{array}{c}\text { Failure } \\
\text { stress }^{\dagger}[\mathrm{MPa}]\end{array}$ & $\begin{array}{c}\text { Experimental } \\
\text { ultimate strain }^{\dagger}\end{array}$ & $\begin{array}{c}\text { Chord modulus of } \\
\text { elasticity }^{+\dagger}[\mathrm{GPa}]\end{array}$ & $\begin{array}{c}\text { Theoretical } \\
\text { ultimate strain }^{*}\end{array}$ \\
\hline Integral specimens (5 samples) & $648.3(11.5 \%)$ & $0.0150(4.8 \%)$ & $44.1(3.5 \%)$ & 0.0147 \\
Peeled specimens (6 samples) & $736.5(6.4 \%)$ & $0.0153(2.5 \%)$ & $51.3(2.1 \%)$ & 0.0143 \\
\hline
\end{tabular}

${ }^{\dagger}$ Terms in parentheses represent the coefficient of variation.

${ }^{+}$According to [5], chord modulus is evaluated as the ratio of stress increment over strain increment, corresponding to the strain interval from 0.001 to 0.003 .

${ }^{*}$ Evaluated as the ratio of the failure stress over the chord modulus of elasticity.

the compression Young's modulus, the following equation was used:

$$
(E I)_{\exp }=E_{\text {tens }} I_{\text {tens }}+E_{\text {comp }} I_{\text {comp }}
$$

in which $(E I)_{\exp }$ is the experimental flexural stiffness, $E_{\text {tens }}$ is tensile Young's modulus obtained from the tensile tests performed on the integral specimens, equal to $44.1 \mathrm{GPa}, I_{\text {tens }}$ and $I_{\text {comp }}$ represent the moment of inertia of the tensile and compression zone, respectively, related to neutral axis, and $E_{\text {comp }}$ is the compression Young's modulus to be evaluated.

The results reveal average global tension and compression Young's moduli of 38.4 and $32.7 \mathrm{GPa}$, respectively, in good agreement with values commonly exhibited by GFRP [6]. These results are used, as clarified in next session, to assess the stress level of the GFRP during the blast tests.

To complete the mechanical characterization, a dynamic tensile characterization campaign was performed, where high strain-rate values of interest ranged from $1 \mathrm{~s}^{-1}$ to $700 \mathrm{~s}^{-1}$. The tests were conducted on the internal core of the composite elements, composed of unidirectional fiber reinforced polymer. The performed tests revealed an increase in tensile strength, which reached $88 \%$ in case of the highest strain-rate. The Young modulus presented an even higher enhancement, increasing up to $178 \%$, in case of the highest strain-rate. A detailed discussion of these characteristics is reported in [7]. The results of the dynamic tensile characterization campaign will be used to construct complete constitutive laws and to validate the dynamic behavior of the barrier system via sophisticated numerical models.

2.2. Blast Tests. Three blast tests were performed on distinct barrier specimens to validate the system's capability to withstand blast-induced loads and reduce the shock wave propagating beyond the barrier. In these tests, a TNT charge was detonated at a specified distance from a specimen composed of 13 pipes, installed as described previously, to form a $2 \mathrm{~m}$ long barrier. Figure 1 shows a specimen before the test. The charge was placed at $1.5 \mathrm{~m}$ from the ground to avoid energy dissipation in the formation of the crater and consequent lack of accuracy in evaluating the quantity of energy propagating in air. In each test, $5 \mathrm{~kg}$ of commercial grade quarry explosive, corresponding to $4 \mathrm{~kg}$ of equivalent TNT explosive was used, and the distances between the charge and the barrier were set at $5 \mathrm{~m}, 3 \mathrm{~m}$, and $0.5 \mathrm{~m}$, respectively. The tests were filmed with high-speed cameras and specimens were instrumented with accelerometers, contact pressure gauges, and strain gauges, located on the central pipe, as depicted in Figure 2, reporting strain gauges labels. Free air pressure gauges were

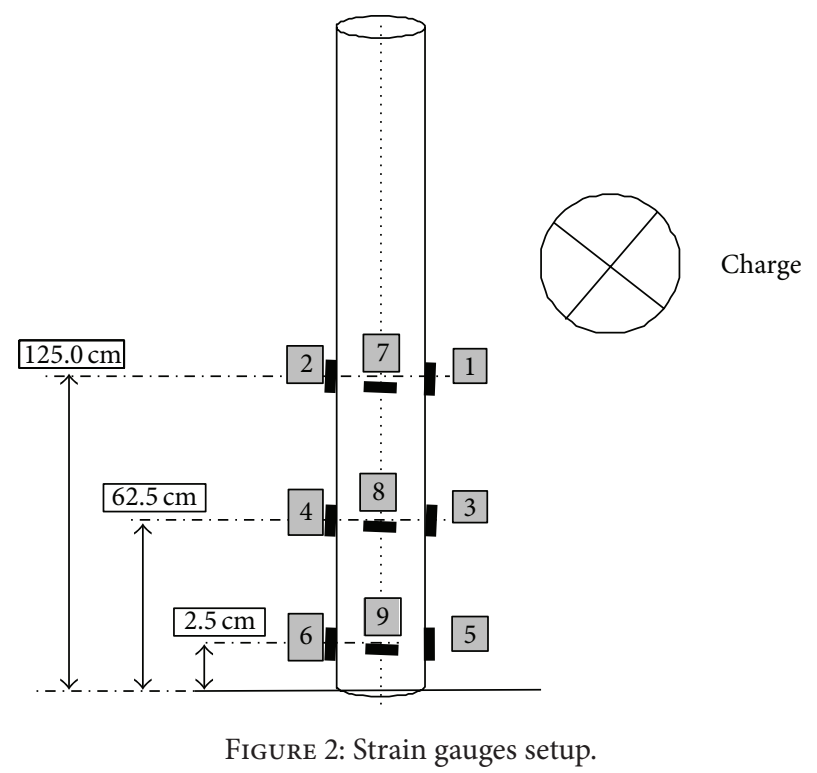

distributed around the barrier to measure the evolution of the pressure field induced by the explosion. The most of the acquired data was only used to validate the blast tests and to assure that results are consistent. However, hereafter only the experimental data useful for the scope of the work are presented.

\section{Results and Discussion}

3.1. Results of the Blast Tests. Strain gauge records allow evaluating the structural response of the central pipe under blast action; such data are available for the first and second blast tests, since damage occurred on the strain gauges during the third test. A preliminary elaboration of strain data is presented, aiming at (a) evaluating the stress levels occurring on instrumented pipes during blast excitation and (b) performing a comparison with levels reached during mechanical static tests.

Maximum absolute strains recorded by strain gauges 1-6 during the first and the second test are reported in Table 2. The largest values were recorded at sensors 5 and 6 , placed close to the base of the pipe; in particular, both sensors recorded the same maximum values of 0.0007 and 0.0019 , during the first and the second test, respectively. These values are much lower than the failure strain obtained in the laboratory for quasi-static tensile tests $(0.0150)$ (Table 1$)$, quasistatic four-point bending tests $(0.0064)$, and high strain-rate 
TABLE 2: Maximum absolute GFRP pipe strains during blast tests.

\begin{tabular}{lcc}
\hline Strain gauge & First shot $[\mathrm{m} / \mathrm{m}]$ & Second shot $[\mathrm{m} / \mathrm{m}]$ \\
\hline 1 & 0.0003 & 0.0014 \\
2 & 0.0003 & 0.0013 \\
3 & 0.0005 & 0.0012 \\
4 & 0.0004 & 0.0013 \\
5 & 0.0007 & 0.0019 \\
6 & 0.0007 & 0.0019 \\
\hline
\end{tabular}

tensile tests (0.0124). Furthermore, the pipes were carefully inspected and no local damage was visually observed. This allows affirming that no structural damage occurred in the GFRP during the first and the second test.

Furthermore, strain data were employed to derive the internal bending moment acting on the instrumented pipe during blast excitation. Onto this end, strain gauges pairs 12, 3-4, and 5-6 (Figure 2) were used. Each of these pairs was placed at a different level and diametrically opposite sides of the pipe element (specifically 125.0, 62.5, and $2.5 \mathrm{~cm}$ from the concrete base, resp.), identifying a cross section. For each pair, the curvature $\chi$ was derived as shown below, assuming that a plane section remains plane under loading:

$$
\chi=\frac{\varepsilon_{1}+\varepsilon_{2}}{h} .
$$

Then, the bending moment history was determined at each instrumented cross section as

$$
M=\chi(E I)_{\exp },
$$

where the average flexural stiffness $(E I)_{\exp }$ obtained from four-point bending tests was used. To conduct such calculation, the quasi-static Young modulus was used, since it was verified, from recorded strain data, that a strain-rate of about $1 \mathrm{~s}^{-1}$ was induced; at this strain-rate value it was verified that the material does not exhibit a significant increase in Young modulus value [8]. Furthermore, the transverse strain gauges measurements (strain gauges 7, 8, and 9 in Figure 2) allowed concluding that ovalization of the cross section could be neglected with respect to its influence on variations of the inertia modulus.

Figures 3 and 4 report bending moment histories at instrumented cross sections for the first and the second test, respectively. In both cases, bending moment histories exhibited sinusoidal trends, clearly induced by oscillating displacements. Maximum values are equal to $0.65 \mathrm{kNm}$ and $1.81 \mathrm{kNm}$ in the first and the second blast, respectively, occurring, in both cases, at the base of the pipe, as it could be expected by a cantilever beam behavior. As further proof of the absence of damage, computed moment values are below $7 \mathrm{kNm}$, representing failure bending moment experienced during four-point bending tests. In both the first and the second blast tests, oscillation started about $330 \mathrm{~ms}$ after explosion time. Considering that the blast pressure reduced to zero not more than $40 \mathrm{~ms}$ after explosion time, as recorded from contact pressure sensors, pipe displacements can be considered as elastic free vibrations, induced by an initial impulsive action.
Figure 5 provides a schematic plan view of the test setup showing the location of the two air pressure gauges S1 and S6, placed at opposite sides with respect to the charge. Gauge S1 measures the free air pressure at a given distance while gauge S6 measures the free air pressure at the same distance from the charge, but behind the barrier. Hence, by comparing the pressure history acquired by these sensors, the interference of the barrier on the shock wave can be directly evaluated. With reference to Figure 5, the distance between gauge 56 and the center of the barrier $\left(D_{T}-D_{B}\right)$ was kept constant at $4 \mathrm{~m}$ for all tests. For this reason, $D_{T}$ was set equal to $9 \mathrm{~m}, 7 \mathrm{~m}$, and $4.5 \mathrm{~m}$ for the first, second, and third blasts, respectively, with $D_{B}$ equal to $5 \mathrm{~m}, 3 \mathrm{~m}$, and $0.5 \mathrm{~m}$. The length of the barrier test article was chosen recognizing that the blast wave beyond the barrier, recorded by rear sensors, is surely affected by diffractions occurring around the barrier. This effect is governed by the height of the barrier, a constant value in this program; hence, specimen width was selected so that the barrier boundaries, both horizontal and vertical, are at the same approximate distance from the charge.

After the first and second tests, the barrier was carefully inspected and no damage was observed, even in the concrete composite interface; the third blast determined the failure of five of the central pipe elements as depicted in Figure 6. Figures 7 to 9 show the pressure-time curves obtained from gauges $\mathrm{S} 1$ and $\mathrm{S} 6$ in each test (data from gauge $\mathrm{S} 1$ in the second test are not available). All diagrams also show the peak pressure estimated according to numerical formulations of Henrych [9] based on the amount of equivalent TNT and the distance of the sensor. These numerical formulations are currently used in the scientific community for free air explosions and are considered reliable. The acquisition of the pressure histories was triggered by the explosion. Therefore, the first portion of the acquired signals reporting zero values represents the delay time as the shock waves traveled to the sensors. As shown in the figures, such time decreases from the first to the third test, as the distance between the explosive and the sensor $D_{T}$ decreases. While the arrival time of the shock wave decreases as the distance $D_{T}$ decreases, the three diagrams show that acquired pressure values increase as distance $D_{T}$ decreases.

Focusing on the barrier's effect in the attenuation of the blast wave's peak pressure, a percentage reduction factor $\eta_{\text {exp }}$, representing the mitigation effect, can be derived from Figures 7 to $9 . \eta_{\exp }$ was evaluated using

$$
\eta_{\exp }=\frac{p_{N B, \exp }-p_{B, \exp }}{p_{N B, \exp }} 100
$$

with $p_{N B \text {,exp }}$ being the peak pressure recorded by gauge $\mathrm{S} 1$ (or derived with the numerical model) and $p_{B, \exp }$ being the peak pressure evaluated and recorded by gauge S6. As a general observation, the maximum reduction factor $\eta_{\exp }$ was obtained for the second test, with a distance between the charge and the barrier of $3 \mathrm{~m}$. This result likely occurs because, in the first test $\left(D_{B}=5 \mathrm{~m}\right)$, the blast wave had more space to grow and surmount the barrier without losing much energy. On the contrary, in the third test $\left(D_{B}=0.5 \mathrm{~m}\right)$, the porosity of the barrier permits the blast wave to pass through 


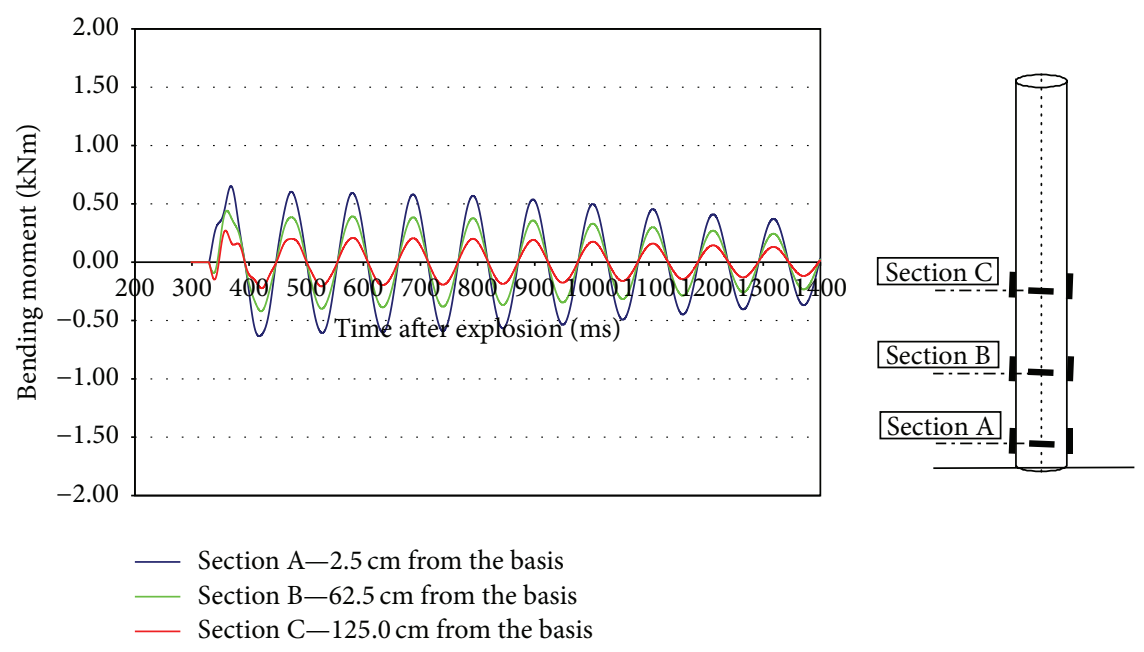

FIGURE 3: Bending histories at instrumented cross sections, first test.

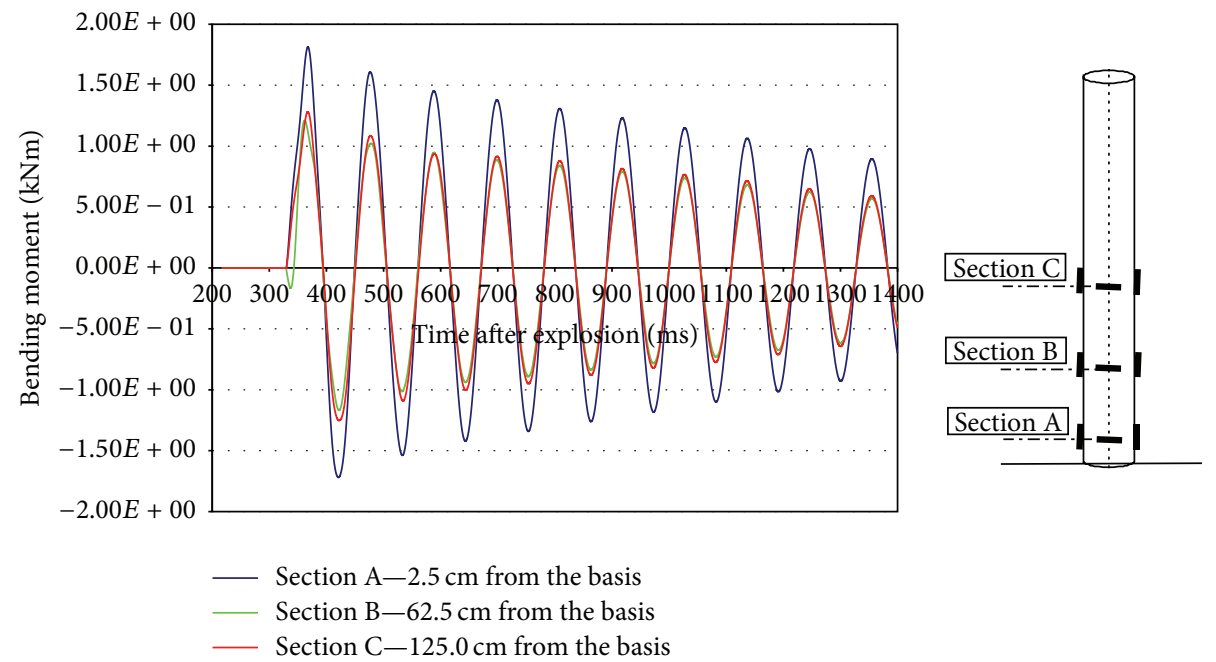

FIGURE 4: Bending histories at instrumented cross sections, second test.

the pipes more easily than in the previous tests. Additionally, in the third test, even if the barrier was seriously damaged, a reduction of the peak pressure was experienced; however it could be argued that the damage to the pipes in the third test could have limited the barrier mitigation effect on the blast wave; unfortunately, no strain gauge data are available for this test to provide an experimental confirmation of this explanation. During each blast test, no crater formation occurred, as desired for the reasons stated above.

3.2. Experimental-Theoretical Comparisons. The availability of these experimental data provides an opportunity to validate a simplified numerical procedure to predict the mitigation effects of a porous barrier. In cases of other potential targets requiring specific protection measures, such procedures could facilitate designing the optimum geometrical configuration of a porous barrier to limit the blast wave to an acceptable value.
Available literature addresses the interaction of shock waves with porous barriers, with many papers focusing on grid configuration [10-13] and stressing the importance of porosity in the fluid-dynamic interaction with shock waves. Other studies investigate the attenuation of different blast waves' impact on a porous barrier [14-16]. Different geometrical configurations of the porous surface were also analyzed, and the interaction with a target placed beyond the barrier was discussed [17-20]. However, differently from continuous barriers, no simple numerical model is available to predict the attenuation of a blast-induced shock wave due to a porous barrier. To this effect, Chapman et al. [21] conducted a blast test campaign, investigating the protection offered by a continuous rigid barrier against a blast-induced shock wave and proposed a simple formulation providing the peak pressure reduction factor. A similar study was conducted by Zhou and Hao [22], who, after an experimental campaign, proposed another numerical formulation. 


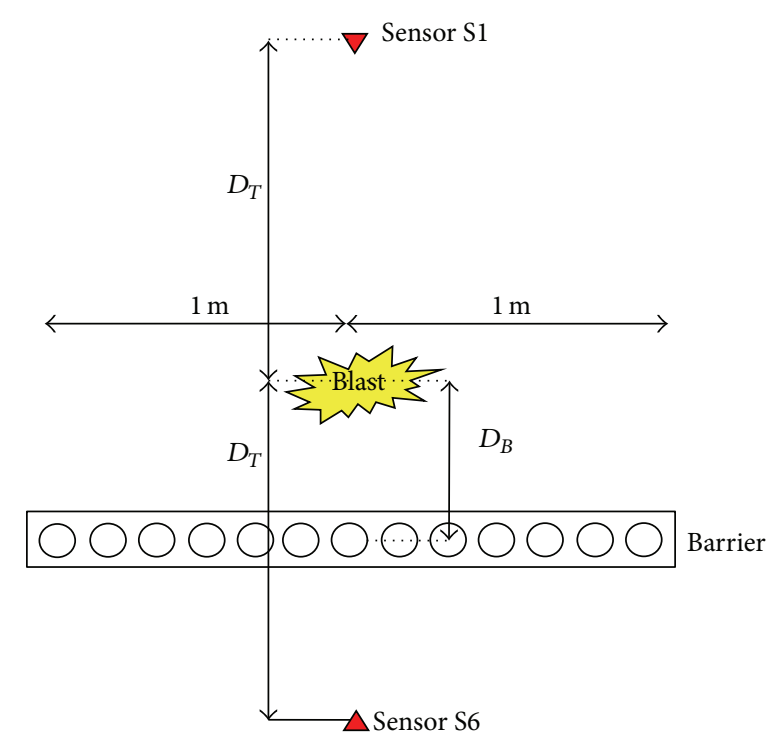

FIGURE 5: Pressure gauges setup.

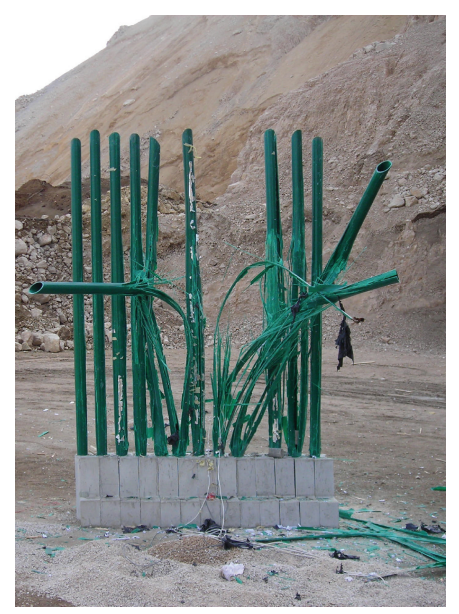

Figure 6: Barrier specimen after the third test.

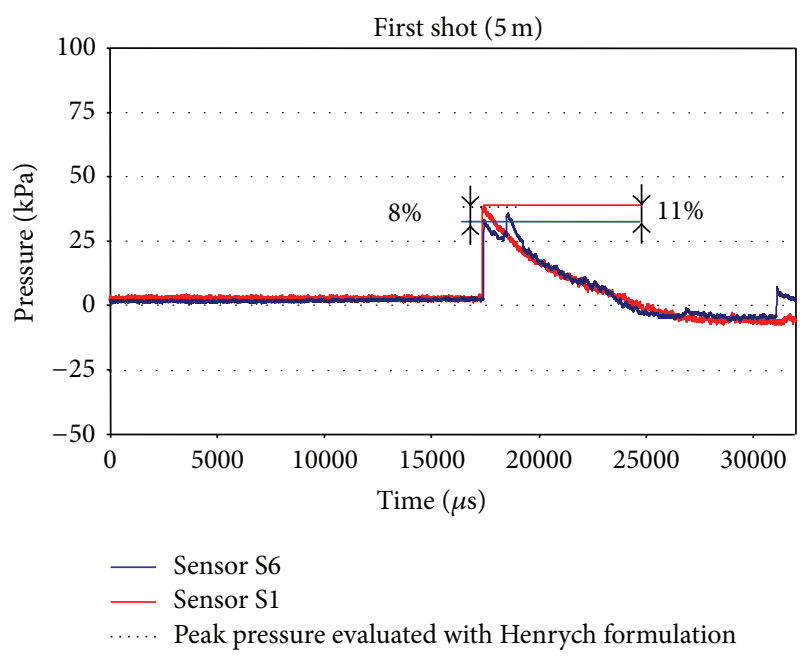

FIgURE 7: Acquired pressure histories: first blast.

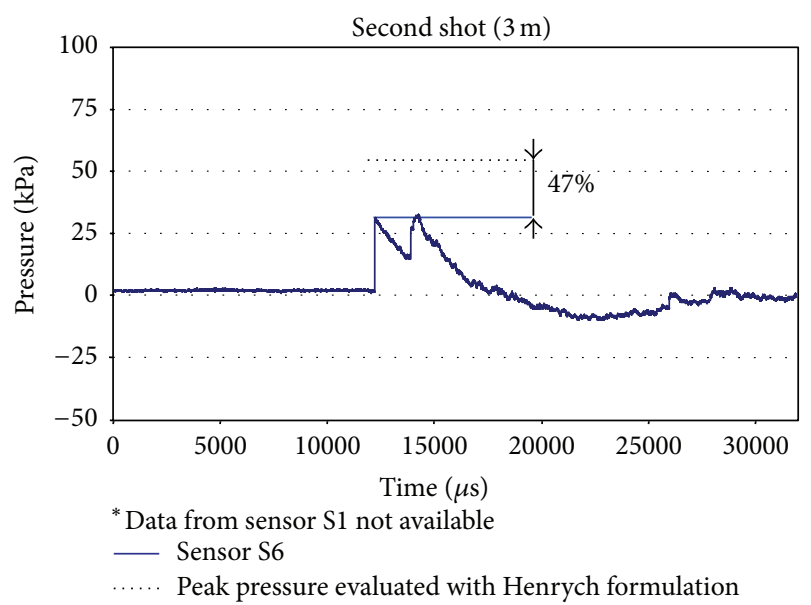

FIGURE 8: Acquired pressure histories: second blast.

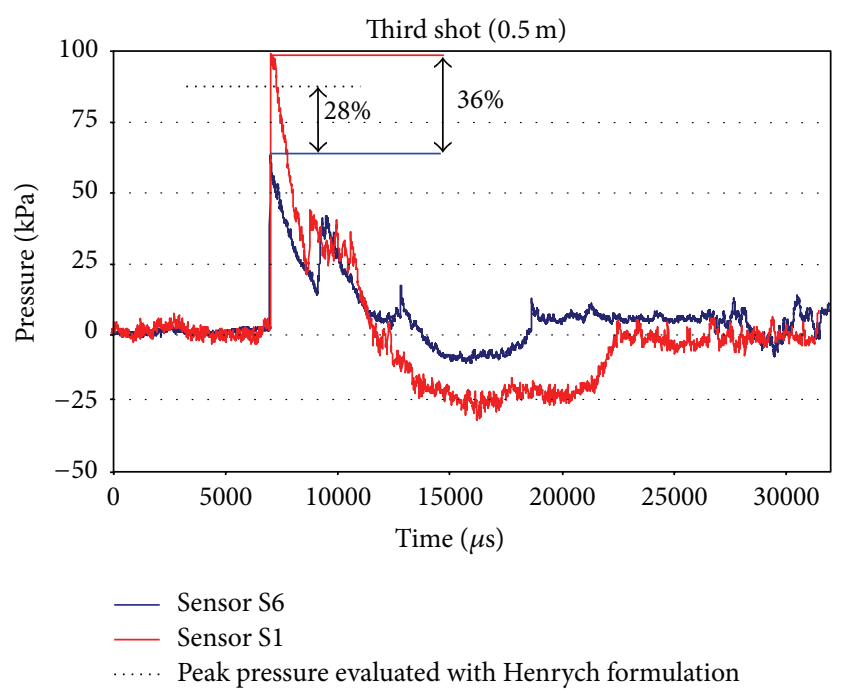

FIgURE 9: Acquired pressure histories: third blast.

Based on available literature, the main parameters governing the wave abatement phenomenon can be identified as follows:

(i) Equivalent TNT charge weight $W$.

(ii) Height of the charge $H_{C}$ from the ground.

(iii) Height of the barrier $H_{B}$.

(iv) Height of the target $H_{T}$.

(v) Distance between the charge and the barrier $D_{B}$.

(vi) Distance between the charge and the target $D_{T}$.

(vii) Porosity of the barrier $r$ computed as the ratio of the voids area over the total frontal area of the barrier.

The proposed procedure does not take into account the material properties of the barrier. This assumption is consistent with the hypothesis used in the analysis of continuous barriers [20, 21]; that is, the barrier is rigid during the first part of the shock wave when the peak pressure is moving 


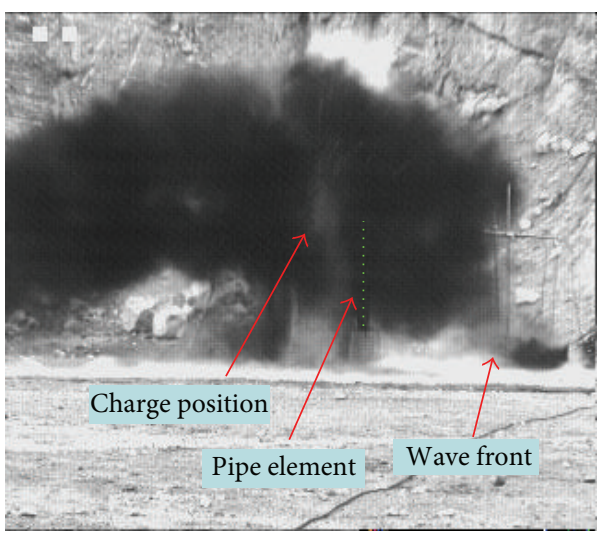

(a)

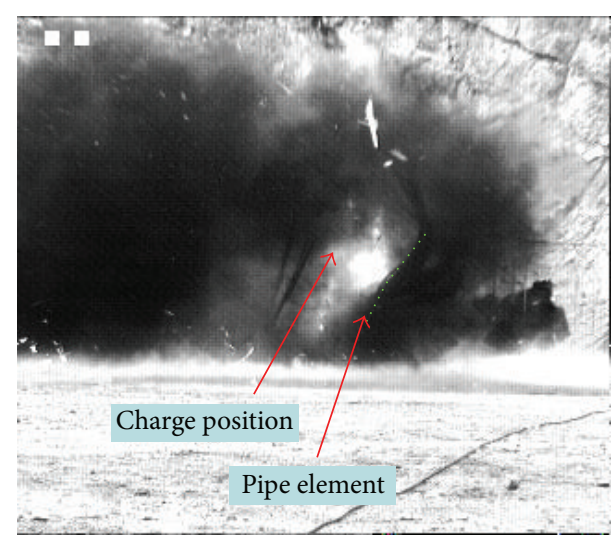

(b)

Figure 10: Acquired frames $2 \mathrm{~ms}$ (a) and $20 \mathrm{~ms}$ (b) after the explosion in the third shot.

throughout it. Since the barrier response is much slower than the shock wave time, as mentioned above, the barrier can be assumed to behave as a rigid body, even though significant damage could occur later, as happened in the third test. Recorded high-speed video confirmed that barrier failure and disaggregation started when the wave front had already overcome the barrier. Indeed, in Figure 10(a), representing the frame acquired $2 \mathrm{~ms}$ after the shot, it can be caught as a glimpse of the barrier, still in its undeformed position, whereas the blast wave front is already beyond the barrier, as it can be guessed looking at the raised dust on the ground. On the contrary, in Figure 10(b), representing the frame acquired $20 \mathrm{~ms}$ after the shot, the barrier in its deformed configuration can be clearly seen. However, this assumption may not be valid in the case of very weak barriers, not capable of withstanding the first part of the shock wave. For these cases, more sophisticated numerical models should be used. The procedure does not account for blast wavelength, but, for the case at hand, this is not detrimental since the ratio blast wavelength-to-barrier porosity is very large and a small change in this ratio would not affect the procedure effectiveness. In particular, the blast wavelength at the barrier was nearly equal to $2 \mathrm{~m}$ in each of the conducted tests. For this reason, the proposed procedure remains valid for blast scenarios in which the ratio blast wavelength-to-barrier porosity is similar to that occurring in the investigated cases. Moreover, the procedure does not account for boundary effects, occurring at the lateral sides of the barrier; actually lateral boundaries should not provide significant effects, since, in the test setup, the top boundary is as far as lateral boundaries from the detonation point and should provide similar interactions with blast waves. More sophisticated models could be developed to account for this effect and remain valid in a wider set of cases.

The proposed simplified procedure allows for evaluating the reduction factor of the peak pressure at a given distance from the charge, because of the interposition of a porous barrier. This procedure, summarized through the steps listed below, is presented in Appendix using the data from the first blast test.
Step 1. The peak pressure $p_{1}$, corresponding to the barrier (Figure 11) at the distance $D_{B}$, due to the detonation of $W$ is evaluated according to the numerical formula [9], since it is a typical free air phenomenon.

Step 2. The blast pressure $p_{1}$ is divided into two components, depending on the porosity of the barrier:

$$
\begin{aligned}
& p_{1 V}=p_{1} \cdot \rho, \\
& p_{1 S}=p_{1} \cdot(1-\rho) .
\end{aligned}
$$

The pressures $p_{1 V}$ and $p_{1 S}$ represent the impacting actions on the voids and the solid parts, respectively.

It is underlined that the porosity of the barrier is not affected by its width, since the barrier is assumed to work in an indefinite configuration, as the case when the target to be protected is fenced by a closed barrier.

Step 3. Working backwards with procedures from the numerical formula [9], two fictitious TNT charges are determined to be $W_{1}$ and $W_{2}$, as the amounts able to generate the peak pressure values $p_{N B 1}$ and $p_{N B 2}$ at the distance $D_{B}$. The sum of the fictitious charges does not correspond to $W$, since pressure does not decrease according to a linear law.

Step 4 . The peak pressure $p_{B \text {,num }}$ on the target is then evaluated as the sum of two contributions $p_{B 1}$ and $p_{B 2}$ :

$$
p_{B, \text { num }}=p_{B V}+p_{B S},
$$

where $p_{B V}$ is the peak pressure generated by the detonation of the fictitious charge $W_{1}$ in free air at the distance $D_{T}$ evaluated according to the numerical model [9] and $p_{B S}$ is the peak pressure generated by the detonation of the fictitious charge $W_{2}$ at the distance $D_{T}$ behind a continuous barrier with the height of the porous barrier evaluated according to procedures found in the literature [20] or [21]. The peak pressure $p_{B V}$ is evaluated accounting for eventual ground reflection, since the numerical model [9] does consider potential interaction with reflected Mach wave, generated below the triple point $[4,9]$. 


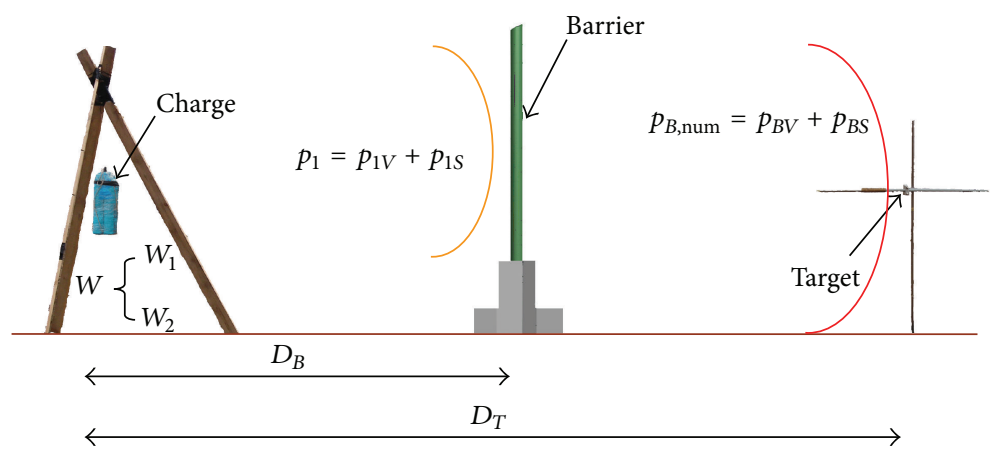

FIGURE 11: Geometrical representation of the proposed model.
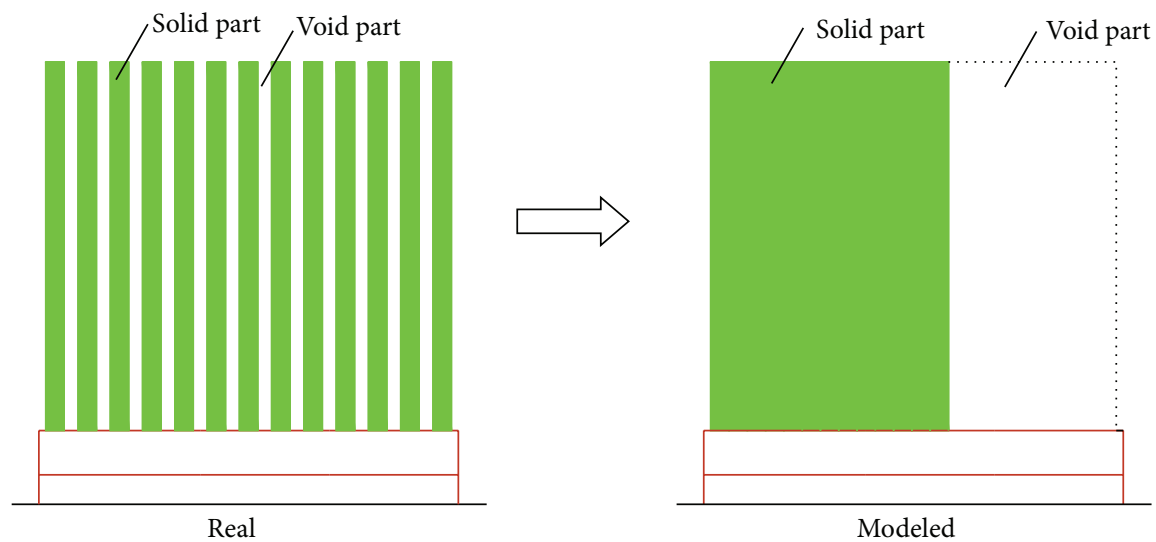

FIGURE 12: Modeling of the barrier.

Step 5. The reduction factor $h_{\text {num }}$ is finally evaluated as

$$
\eta_{\text {num }}=\frac{p_{N B, \text { num }}-p_{B, \text { num }}}{p_{N B, \text { num }}} \cdot 100,
$$

where $p_{N B \text {,num }}$ is the pressure generated by the charge $W$ in free air at the distance $D_{T}$ evaluated according to the numerical model [9].

The proposed simplified model neglects the fluiddynamic turbulence close to the pipes, assuming that the barrier could be equivalently reduced into a void and a solid part, as shown in Figure 12. It is underlined that this does not mean that the porous barrier is equivalent to smaller solid one; in fact the peak pressure reduction is mainly due to the diffraction of the shock wave passing through the pores. Hence, the scheme in Figure 12 is only used in the simplified numerical procedure. The described procedure was applied to the configuration used in the blast tests; in this case, the porosity $\rho$ of the barrier, evaluated as the ratio of the voids over the spacing of the pipe elements, equals 0.43 . In Step 4 , the Zhou and Hao model [22] was used. Table 3 summarizes both experimental and numerical results in terms of free air peak pressure and behind the barrier. For the numerical peak pressure in free air, both Henrych formulations [9], $p_{N B \text { exp }}$, and UFC (Unified Facilities Criteria) formulations $[4], p_{N B \text {,exp,UFC }}$, were used, revealing being in good agreement. Table 4 reports the reduction factors evaluated using both experimental and numerical results. In particular, $h_{\exp }$ and $h_{\text {num }}$ values are reported. A hybrid reduction factor $h_{\text {hyb }}$, evaluated using $p_{B \text {,num }}$ against $p_{N B \text {,exp }}$ values, is also presented, and its expression is as follows:

$$
\eta_{\text {hyb }}=\frac{p_{N B, \exp }-p_{B, \text { num }}}{p_{N B, \exp }} \cdot 100 .
$$

It can be observed that free air peak pressure for the second test is not available. However, the numerical reduction factor was also derived, given the reliability of the free air numerical formulations used [9]. When experimental and analytical results are compared, good agreement is observed. In order to quantify completely the blast action, the total impulse (i.e., area below the pressure curve) was also considered. Values recorded during the tests are presented in Table 5. The total impulse reduction factors of $6 \%$ and $25 \%$ were experienced in first and third experiments, respectively, whereas, in second test unavailability of data from sensor S1 does not allow performing such comparison. As depicted in Figures 7-9, pressure histories are characterized by a nonmonotonic decreasing trend, presenting more peak values, which increase total impulse; this can be attributed to both multiple ground reflections and wave diffractions occurring at the boundaries. It is underlined that the procedure applies to the first peak, which can be attributed to the direct blast wave. Nevertheless, the barrier provides a reduction of total impulse, which, as for peak pressures, is larger in the third 
TABLE 3: Experimental and theoretical peak pressure.

\begin{tabular}{|c|c|c|c|c|c|c|}
\hline & \multicolumn{3}{|c|}{ Pressure without the barrier } & \multicolumn{3}{|c|}{ Pressure behind the barrier } \\
\hline & Experimental & Theoretical & Theoretical & Experimental & Theoretical $p_{B}$ & num $[\mathrm{kPa}]$ \\
\hline & $p_{N B, \exp }[\mathrm{kPa}]$ & $p_{N B, \text { num }, \mathrm{UFC}}[\mathrm{kPa}]$ & $p_{N B, \text { num }}[\mathrm{kPa}]$ & $p_{B, \exp }[\mathrm{kPa}]$ & Zhou and Hao model [22] & Chapman model [21] \\
\hline $1^{\circ}$ shot $(5 \mathrm{~m})$ & 37 & 39 & 36 & 33 & 33 & 24 \\
\hline $2^{\circ} \operatorname{shot}(3 \mathrm{~m})$ & Not available & 61 & 58 & 31 & 31 & 28 \\
\hline $3^{\circ} \operatorname{shot}(0.5 \mathrm{~m})$ & 98 & 94 & 88 & 63 & 57 & 64 \\
\hline
\end{tabular}

TABLE 4: Experimental and theoretical peak pressure reduction factors.

\begin{tabular}{lccc}
\hline & $\begin{array}{c}\text { Experimental } \\
h_{\exp }\left(p_{B, \exp } \text { to }\right. \\
\left.p_{N B, \exp }\right)\end{array}$ & $\begin{array}{c}\text { Theoretical } \\
h_{\text {num }}\left(p_{B, \text { num }}\right. \\
\left.p_{N B, \text { num }}\right)\end{array}$ & $\begin{array}{c}\text { Hybrid } \\
h_{\text {hyb }}\left(p_{B, \text { num }}\right. \\
\left.p_{N B, \exp }\right)\end{array}$ \\
\hline $1^{\circ}$ shot $(5 \mathrm{~m})$ & $11 \%$ & $8 \%$ & $11 \%$ \\
$2^{\circ}$ shot $(3 \mathrm{~m})$ & Not available & $47 \%$ & Not available \\
$3^{\circ}$ shot $(0.5 \mathrm{~m})$ & $36 \%$ & $35 \%$ & $42 \%$ \\
\hline
\end{tabular}

TABLE 5: Experimental total impulse and relative reduction factors.

\begin{tabular}{lccc}
\hline & $\begin{array}{c}\text { Total impulse } \\
\text { without the } \\
\text { barrier }[\mathrm{kPa} \cdot \mathrm{ms}]\end{array}$ & $\begin{array}{c}\text { Total impulse } \\
\text { behind the } \\
\text { barrier }[\mathrm{kPa} \cdot \mathrm{ms}]\end{array}$ & $\begin{array}{c}\text { Reduction } \\
\text { factor }\end{array}$ \\
\hline $1^{\circ}$ shot $(5 \mathrm{~m})$ & 99.9 & 93.9 & $6 \%$ \\
$2^{\circ}$ shot $(3 \mathrm{~m})$ & Not available & 85.2 & Not available \\
$3^{\circ}$ shot $(0.5 \mathrm{~m})$ & 167.4 & 133.9 & $25 \%$ \\
\hline
\end{tabular}

test than in the first one. A theoretical evaluation of impulse at sensor $\mathrm{S} 1$ in the second test is not performed here since such evaluation would be quite uncertain due to the difficulty of predicting such spurious contributions. It is underlined that the numerical procedure used to predict the reduction factor does not depend on width of the barrier, which actually does not affect the reduction factor, whereas the height is accounted in the Zhou and Hao method [22] used in the procedure.

\section{Conclusions}

Results of a blast test campaign conducted on a blast protection porous barrier composed of GFRP pipe elements mounted on a precast, GFRP-reinforced concrete foundation were presented. The results show the capability of the barrier in disrupting the shock wave generated by an explosion and consequently reducing the induced loads on a target placed beyond the barrier.

As the distance between a detonating charge and the barrier decreased from the first to the third blast, the effectiveness in attenuating the blast wave, evaluated through the reduction factor $h_{\text {exp }}$, increased from the first to the second test and then decreased in the third test. This result probably occurs because of the two opposite mechanisms related to such distances. In general, as the charge gets closer to the barrier, a smaller blast sphere impacts the barrier as less space is available for it to develop; consequently, the blast wave cannot surmount the barrier and dissipates a greater portion of its energy to pass beyond it. On the contrary, for small distances, the barrier porosity effect probably becomes more significant, and the blast wave transits more easily through the voids. In order to enrich the experimental research, additional test configurations will be numerically investigated. In fact, future work will include the development of a numerical model of the test, in order to conduct simplified fluid-dynamic analyses. The objective is to validate the proposed procedure for the evaluation of the blast wave reduction, focusing attention on the following issues:

(i) Understanding the actual phenomenon evolution, discerning between the boundary effects, and the actual wave diffraction due to the porous barrier.

(ii) Simulating additional test configurations with different charge weights and locations.

A simplified model predicting the reduction effect of the porous barrier on the peak pressure is proposed following the protocol used for a continuous barrier. The proposed model was validated using the experimental data obtained in the experimental campaign and showed good predictions. The model cannot capture complicated fluid-dynamic phenomena occurring close to the barrier; however, the discrepancies between numerical and experimental results appear to be below the uncertainty related to the evaluation of a blastinduced pressure.

\section{Appendix}

The proposed simplified model is here applied to the first test configuration. In such case, the input parameters assume the following values:

(i) Equivalent TNT charge weight $W=4 \mathrm{~kg}$.

(ii) Height of the charge from the ground $H_{C}=1.5 \mathrm{~m}$.

(iii) Height of the barrier $H_{B}=3.0 \mathrm{~m}$.

(iv) Height of the target $H_{T}=1.5 \mathrm{~m}$.

(v) Distance between the charge and the barrier $D_{B}=$ $5.0 \mathrm{~m}$.

(vi) Distance between the charge and the target $D_{T}=$ $9.0 \mathrm{~m}$.

(vii) Porosity of the barrier $\rho=0.43$. 
Step 1. According to the numerical model [9], the free air pressure at the distance $D_{B}$ can be evaluated as follows:

$$
\begin{aligned}
p_{1} & =\left(\frac{0.662}{\bar{R}}+\frac{4.05}{\bar{R}^{2}}+\frac{3.288}{\bar{R}^{3}}\right) k_{R}=1.15 \mathrm{Kgf} / \mathrm{cm}^{2} \\
& =115 \mathrm{KPa}
\end{aligned}
$$

having obtained the scaled distance $\bar{R}$ as

$$
\bar{R}=\frac{D_{B}}{W^{1 / 3}}=\frac{5.0}{\sqrt[3]{4}}=3.14 \mathrm{~m} / \mathrm{Kg}^{1 / 3},
$$

where $k_{R}$ accounts for the ground reflected pressure and depends on the geometry and the intensity of the blast. It is set equal to 1.6, as suggested in the numerical model [9].

Step 2. Based on the porosity of the barrier,

$$
\begin{aligned}
& p_{1 V}=p_{1} \cdot \rho=115 \cdot 0.43=50 \mathrm{KPa}, \\
& p_{1 S}=p_{1} \cdot(1-\rho)=115 \cdot(1-0.43)=65 \mathrm{KPa} .
\end{aligned}
$$

Step 3. Working backwards from the procedure for free air pressure calculation, with few iterations, the fictitious TNT amounts $W_{1}$ and $W_{2}$ are obtained:

$$
\begin{aligned}
& W_{1}=0.93 \mathrm{Kg}, \\
& W_{2}=1.47 \mathrm{Kg} .
\end{aligned}
$$

Step 4. Amount $W_{1}$ is used to evaluate the free air pressure $p_{B V}$ at the distance $D_{T}$ :

$$
\begin{aligned}
\bar{R} & =\frac{D}{W^{1 / 3}}=\frac{9}{\sqrt[3]{0.93}}=9.22 \mathrm{~m} / \mathrm{Kg}^{1 / 3}, \\
k_{R} & =1.6 \\
p_{B V} & =\left(\frac{0.662}{\bar{R}}+\frac{4.05}{\bar{R}^{2}}+\frac{3.288}{\bar{R}^{3}}\right) k_{R}=0.19 \\
& =19 \mathrm{KPa} .
\end{aligned}
$$

The Zhou and Hao model [22] is used to evaluate the pressure $p_{B S}$ generated by the charge $W_{2}$ at the distance $D_{T}$, behind a rigid continuous barrier. Consider

$$
p_{B S}=p_{B S F} * A_{p},
$$

where $p_{B S F}$ represents the pressure generated by the same charge at the same distance without the barrier, here evaluated using the model from [9], and $A_{p}$ is given as follows:

$$
\begin{aligned}
A_{P}= & -0.1359+\left(0.3272+0.1995 \lg \left(\frac{H_{B}}{D_{T}}\right)\right) \lg \bar{R} \\
& -0.5626 \lg \left(\frac{H_{B}}{D_{T}}\right)+0.4666\left(\frac{D_{B}}{D_{T}}\right)
\end{aligned}
$$

and therefore

$$
\begin{aligned}
p_{B S F} & =24 \mathrm{kPa}, \\
A_{p} & =0.60, \\
p_{B S} & =p_{B S F} * A_{p}=24 * 0.60=14 \mathrm{kPa} .
\end{aligned}
$$

The reduced pressure is evaluated as shown below and is equal to $33 \mathrm{KPa}$ :

$$
p_{B, \text { num }}=p_{B V}+p_{B S}
$$

Step 5. According to the numerical model [9], the free air pressure generated by $W$ at the distance $D_{T}$ without the barrier can be evaluated as

$$
\begin{aligned}
p_{N B, \text { num }} & =\left(\frac{0.662}{\bar{R}}+\frac{4.05}{\bar{R}^{2}}+\frac{3.288}{\bar{R}^{3}}\right) k_{R} \\
& =0.36 \mathrm{Kgf} / \mathrm{cm}^{2}=36 \mathrm{KPa}
\end{aligned}
$$

since

$$
\bar{R}=\frac{D_{T}}{W^{1 / 3}}=\frac{9}{\sqrt[3]{4}}=5.67 \mathrm{~m} / \mathrm{Kg}^{1 / 3}
$$

and, in this case,

$$
k_{R}=1.4
$$

and then the numerical reduction factor $h_{\text {num }}$ can be evaluated as given below and is equal to 8 percent:

$$
\eta_{\text {num }}=\frac{p_{N B, \text { num }}-p_{B, \text { num }}}{p_{N B, \text { num }}} \cdot 100
$$

\section{Conflict of Interests}

The authors declare that there is no conflict of interests regarding the publication of this paper.

\section{Acknowledgment}

The authors gratefully thank European Commission Directorate General Justice, Freedom and Security for the financial support through EPCIP 2006.

\section{References}

[1] D. Asprone, D. Assante, A. Chiariello et al., "Case study: assessment of the electromagnetic disturbance of a glass fiber reinforced composite fencing structure," ASCE Journal of Composites For Construction, vol. 14, pp. 629-635, 2010.

[2] W. G. Corley, M. A. Sozen, C. H. Thornton, and P. F. Mlakar, "The Oklahoma City bombing: improving building performance through multi-hazard mitigation," Tech. Rep. FEMA277, Mitigation Directorate, U.S. Government Printing Office, Washington, DC, USA, 1996.

[3] L. J. Malvar, J. E. Crawford, and K. B. Morrill, "Use of composites to resist blast," Journal of Composites for Construction, vol. 11, no. 6, pp. 601-610, 2007.

[4] US Departments of the Army-the Navy and the Air Force, UFC 3-340-02 Structures to Resist the Effects of Accidental Explosions, US Departments of the Army, the Navy and the Air Force, 2008.

[5] D. Asprone, A. Nanni, H. Salem, and H. Tagel-Din, "Applied element method analysis of porous GFRP barrier subjected to blast," Advances in Structural Engineering, vol. 13, no. 1, pp. 153$169,2010$. 
[6] J. G. Teng, J. F. Chen, S. T. Smith, and L. Lam, FRP: Strengthened RC Structures, John Wiley \& Sons, 2002.

[7] D. Asprone, E. Cadoni, A. Prota, and G. Manfredi, "Strain-rate sensitivity of a pultruded E-glass/polyester composite," Journal of Composites for Construction, vol. 13, no. 6, pp. 558-564, 2009.

[8] ASTM, "Standard test method for tensile properties of polymer matrix composite materials," ASTM D 3039/D 3039M, 2003.

[9] J. Henrych, The Dynamics of Explosion and Its Use, Elsevier, 1979.

[10] A. Britan, O. Igra, G. Ben-Dor, and H. Shapiro, "Shock wave attenuation by grids and orifice plates," Shock Waves, vol. 16, no. 1, pp. 1-15, 2006.

[11] D. S. Dosanjh, "Interaction of grids with traveling shock waves," NACA TN 3680, 1956.

[12] W. J. Franks, "Interaction of a shock wave with a wire screen," UTIA TN 13, 1957.

[13] B. E. Gelfand, S. P. Medvedev, A. N. Polenov, and S. M. Frolov, "Interaction of non-stationary pressure waves with perforated partitions," Archivum Combustionis, vol. 7, pp. 215-222, 1987.

[14] O. Igra, X. Wu, J. Falcovitz, T. Meguro, K. Takayama, and W. Heilig, "Experimental and theoretical study of shock wave propagation through double-bend ducts," Journal of Fluid Mechanics, vol. 437, pp. 255-282, 2001.

[15] O. Igra, X. Wu, G. Q. Hu, and J. Falcovitz, "Shock wave propagation into a dust-gas suspension inside a double-bend conduit," Journal of Fluids Engineering, vol. 124, no. 2, pp. 483491, 2002.

[16] A. Sasoh, K. Matsuoka, K. Nakashio et al., "Attenuation of weak shock waves along pseudo-perforated walls," Shock Waves, vol. 8, no. 3, pp. 149-159, 1998.

[17] B. W. Skews and K. Takayama, "Flow through a perforated surface due to shock-wave impact," Journal of Fluid Mechanics, vol. 314, pp. 27-52, 1996.

[18] G. J. Ball and R. A. East, "Shock and blast attenuation by aqueous foam barriers: influences of barrier geometry," Shock Waves, vol. 9, no. 1, pp. 37-47, 1999.

[19] B. W. Skews, M. D. Atkins, and M. W. Seitz, "The impact of a shock wave on porous compressible foams," Journal of Fluid Mechanics, vol. 253, no. 1, pp. 245-265, 1993.

[20] H. Naiman and D. D. Knight, "The effect of porosity on shock interaction with a rigid, porous barrier," Shock Waves, vol. 16, no. 4-5, pp. 321-337, 2007.

[21] T. C. Chapman, T. A. Rose, and P. D. Smith, "Reflected blast wave resultants behind cantilever walls: a new prediction technique," International Journal of Impact Engineering, vol. 16, no. 3, pp. 397-403, 1995.

[22] X. Q. Zhou and H. Hao, "Prediction of airblast loads on structures behind a protective barrier," International Journal of Impact Engineering, vol. 35, no. 5, pp. 363-375, 2008. 

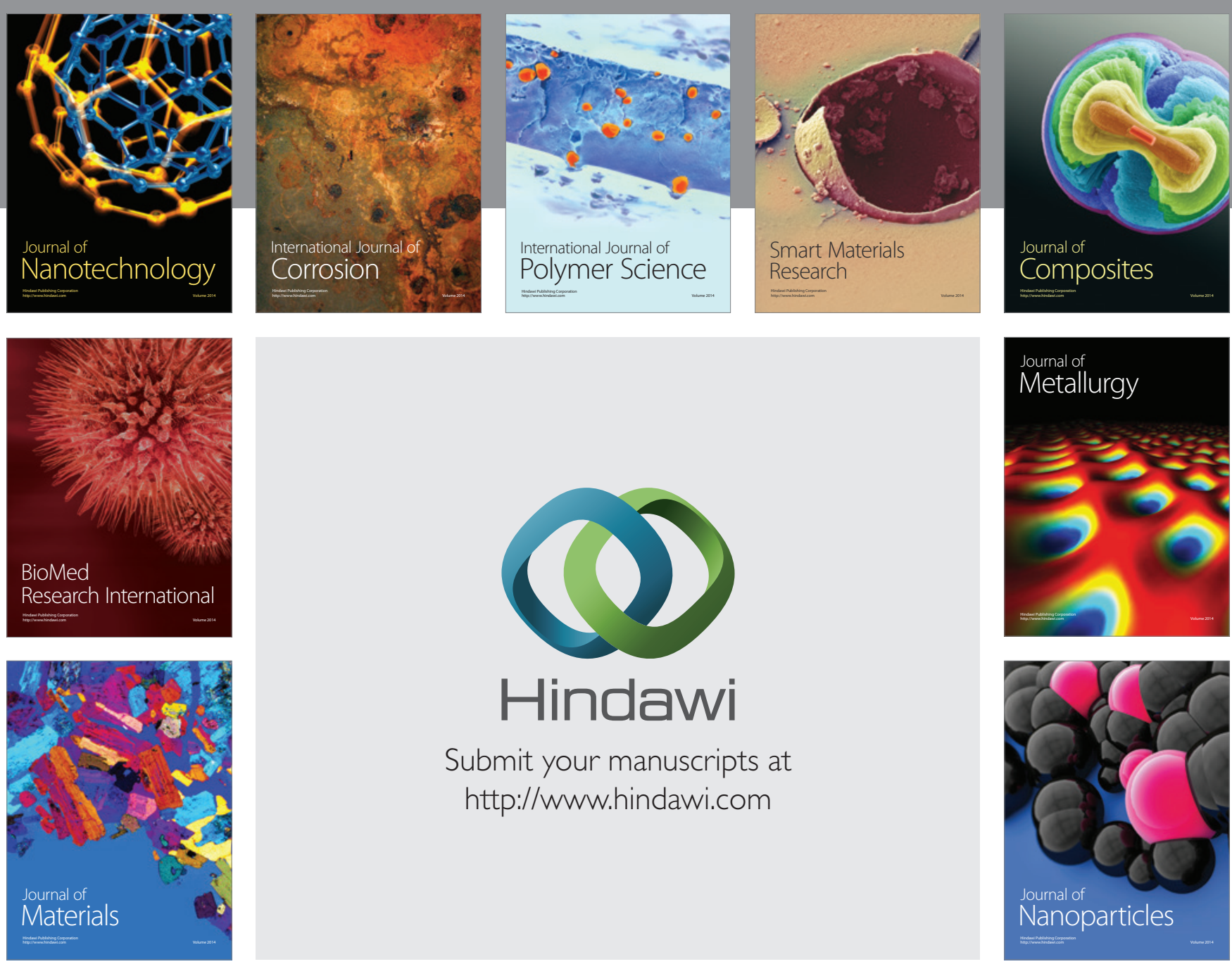

Submit your manuscripts at http://www.hindawi.com
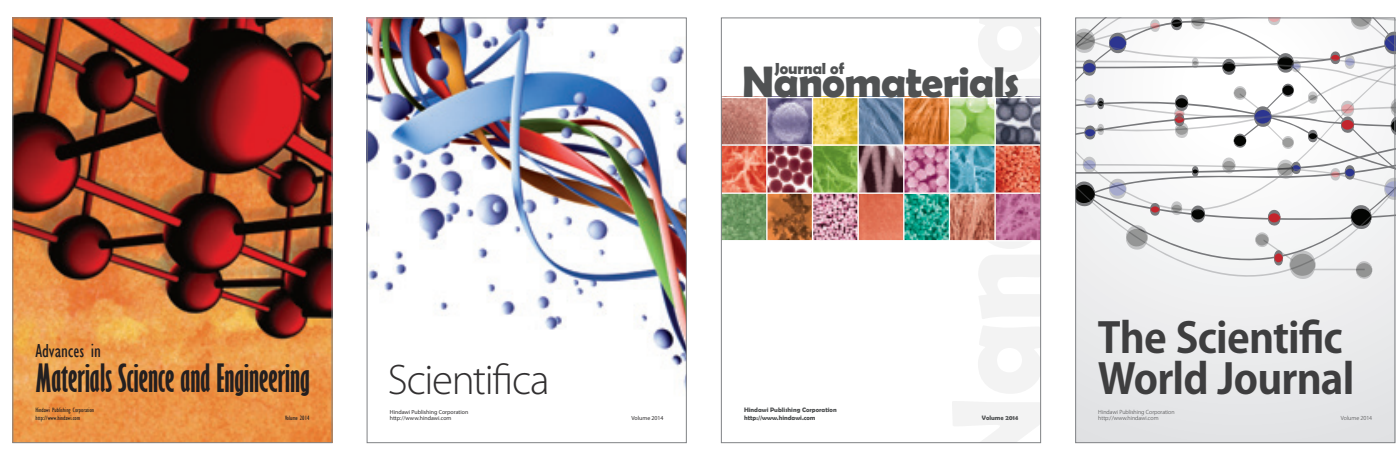

\section{The Scientific World Journal}
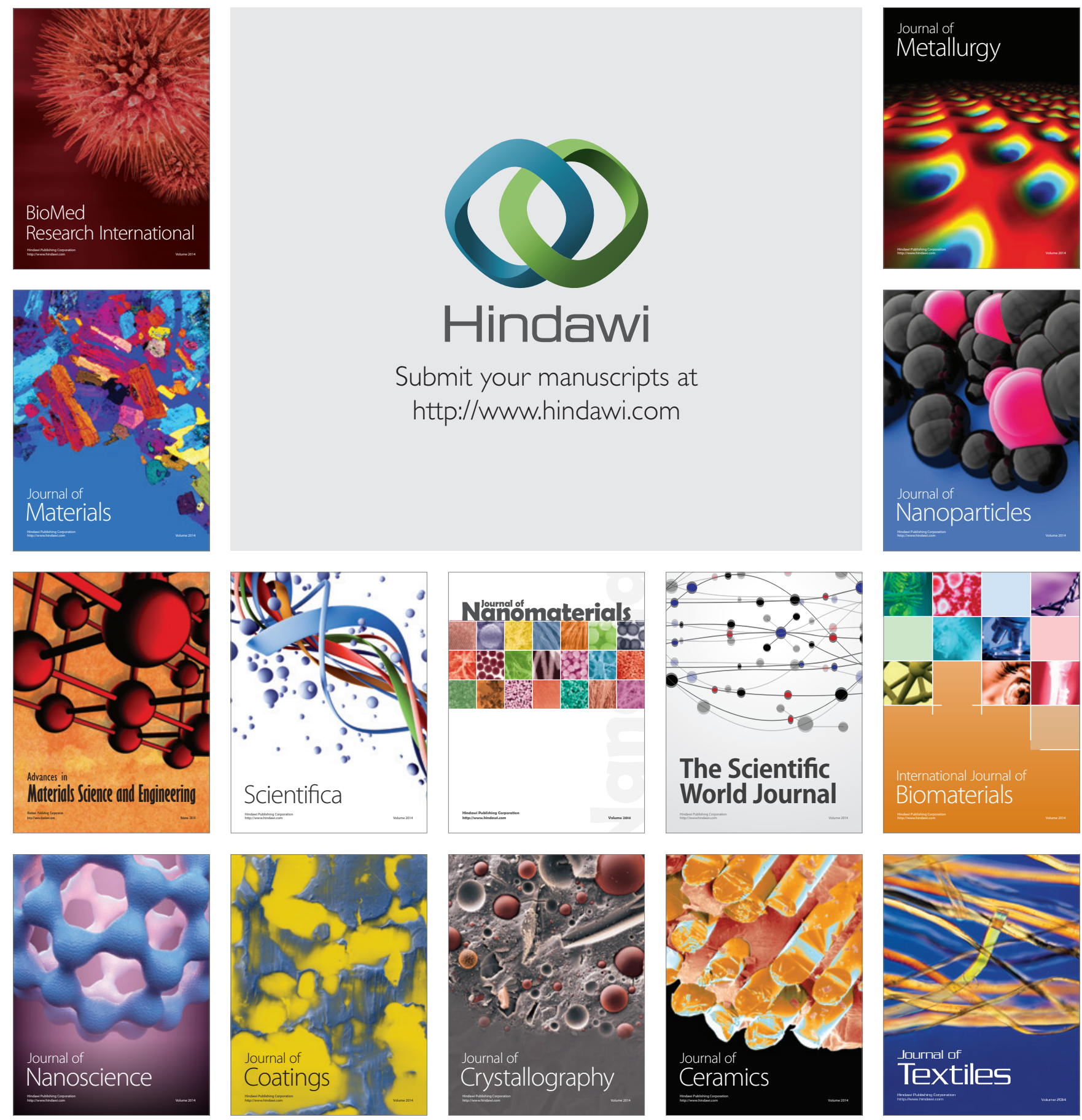